\title{
Cortical Reorganization after Long-Term Adaptation to Retinal Lesions in Humans
}

\author{
Susana T. L. Chung \\ School of Optometry, University of California, Berkeley, Berkeley, California 94720-2020
}

\begin{abstract}
Single-unit recordings demonstrated that the adult mammalian visual cortex is capable of reorganizing after induced retinal lesions. In humans, whether the adult cortex is capable of reorganizing has only been studied using functional magnetic resonance imaging, with equivocal results. Here, we exploited the phenomenon of visual crowding, a major limitation on object recognition, to show that, in humans with long-standing retinal (macular) lesions that afflict the fovea and thus use their peripheral vision exclusively, the signature properties of crowding are distinctly different from those of the normal periphery. Crowding refers to the inability to recognize objects when the object spacing is smaller than the critical spacing. Critical spacing depends only on the retinal location of the object, scales linearly with its distance from the fovea, and is approximately two times larger in the radial than the tangential direction with respect to the fovea, thus demonstrating the signature radial-tangential anisotropy of the crowding zone. Using retinal imaging combined with behavioral measurements, we mapped out the crowding zone at the precise peripheral retinal locations adopted by individuals with macular lesions as the new visual reference loci. At these loci, the critical spacings are substantially smaller along the radial direction than expected based on the normal periphery, resulting in a lower scaling of critical spacing with the eccentricity of the peripheral locus and a loss in the signature radial-tangential anisotropy of the crowding zone. These results imply a fundamental difference in the substrate of cortical processing in object recognition following long-term adaptation to macular lesions.
\end{abstract}

\section{Introduction}

To see an object clearly, most of us effortlessly direct the part of our retina where vision is the most acute, the fovea, toward the object. When there are retinal lesions that ablate the foveal area in both eyes, humans with the condition must place a visual object outside the lesioned foveal region, i.e., in the peripheral retina. The dogma is that visual processing for these individuals who must use their peripheral retina is the same as that in the normal periphery. Here, we exploit the phenomenon of visual crowding, which has been established as a major limiting factor on object recognition in the peripheral retina (Levi, 2008; Pelli, 2008), to show that there is a novel type of cortical plasticity and reorganization that follows the longterm adaptation to foveal lesions, even for people in their eighth decade of life.

Crowding refers to the impaired ability to recognize an object in clutter. It is a cortical phenomenon (Flom et al., 1963) and occurs when an object and its nearest neighbor (flanker) fall within the same region in which integration of visual information occurs (Levi, 2008; Pelli, 2008). To overcome crowding, the ob-

Received June 28, 2013; revised Sept. 16, 2013; accepted 0ct. 8, 2013.

Author contributions: S.C. designed research; S.C. performed research; S.C. contributed unpublished reagents/ analytic tools; S.C. analyzed data; S.C. wrote the paper.

This work was supported by National Institutes of Health/National Eye Institute Grant R01-EY012810. I thank Yiji Lin for technical support, Girish Kumar for assistance with data presentation, and Jean-Baptiste Bernard, Dennis Levi, Saumil Patel, and Bosco Tjan for their helpful comments on a previous version of this manuscript.

The author declares no competing financial interests.

Correspondence should be addressed to Susana T. L. Chung, School of Optometry, University of California, Berkeley, Berkeley, CA 94720-2020. E-mail: s.chung@berkeley.edu.

DOI:10.1523/JNEUROSCI.2764-13.2013

Copyright $\odot 2013$ the authors $\quad 0270-6474 / 13 / 3318080-07 \$ 15.00 / 0$ ject must therefore be separated from its flanker by a distance greater than the dimension of the integration zone, the critical spacing. There are several well known hallmarks of critical spacing. At the retinal level, it scales with eccentricity (Bouma's law) and is always larger along the radial direction toward the fovea than the tangential direction (Bouma, 1970; Toet and Levi, 1992), thus giving rise to the signature radial-tangential anisotropy. The scaling factor of critical spacing with eccentricity is approximately $0.4-0.5$ times radially and approximately $0.1-0.2$ times tangentially (Chung et al., 2001). Readers can experience this signature radial-tangential anisotropy of peripheral crowding in Figure 1. For the same separation between the flanker and the target (Fig. $1 a, b)$, readers should find it easier to see the middle target letter $\mathrm{H}$ in $a$ when the two flankers $\mathrm{R}$ and $\mathrm{S}$ are tangential to fixation than in $b$ when the two flankers $\mathrm{N}$ and $\mathrm{K}$ are radial to fixation.

Critical spacing represents the neuroanatomical limit of cortical processing of object recognition (Pelli, 2008; Pelli and Tillman, 2008), because it is determined solely by the location of the object in the peripheral retina. For people with macular lesions who have to rely on a peripheral retinal location for seeing, it is assumed that the critical spacing measured at the peripheral location would be the same as that in the normal periphery, preserving the signature radial-tangential anisotropy of the crowding zone. Surprisingly, we found a loss in the radial-tangential anisotropy of the two-dimensional crowding zone for people who have adapted to their macular lesions, such that the shape of these crowding zones resembles that of the normal fovea (isotropic in shape). This change in the shape of the crowding zone cannot be explained by a general improvement of visual recogni- 
a
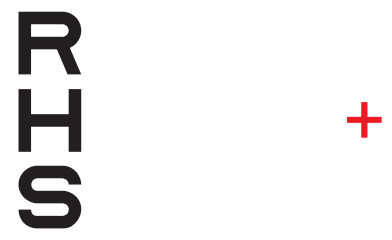

b

NHK

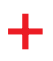

C
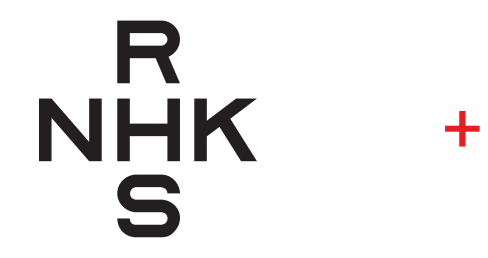

d

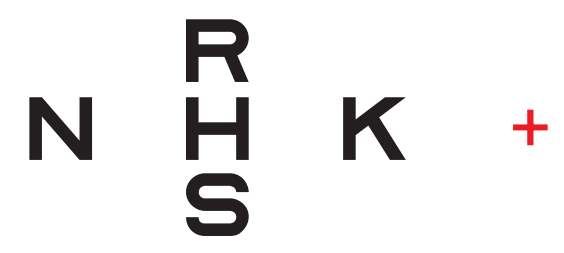

Figure 1. While fixating the red cross in $\boldsymbol{a}$, adjust the viewing distance until you can barely recognize the letter $\mathrm{H}$ on the left of the red fixation cross. The two flanking letters $\mathrm{R}$ and $\mathrm{S}$ are above and below the letter $H$, and the three letters form a line that is tangential with respect to fixation (presumably where your fovea is). Now shift your fixation to $\boldsymbol{b}$, you should find it impossible to recognize the letter $\mathrm{H}$, despite that the letter $\mathrm{H}$ is at the same distance with respect to the fixation cross as in $\boldsymbol{a}$. In this case, the two flanking letters $\mathrm{N}$ and $\mathrm{K}$ are the same distance from $\mathrm{H}$, as $\mathrm{R}$ and $\mathrm{S}$ are in $\boldsymbol{a}$; however, when the letters $\mathrm{N}, \mathrm{H}$, and $\mathrm{K}$ form a line that is radial to fixation, this distance is not sufficient to allow $H$ to be perceived clearly. c shows that, when four flanking letters $\mathrm{R}, \mathrm{K}, \mathrm{S}$, and $\mathrm{N}$ are separated from $\mathrm{H}$ by the same distance, the letter $\mathrm{H}$ cannot be perceived clearly; however, when the two flanking letters that are radial with respect to the fovea ( $\mathrm{N}$ and $\mathrm{K}$ ) are separated from $\mathrm{H}$ by a larger distance, then the letter $\mathrm{H}$ can be perceived clearly $(\boldsymbol{d})$. $\boldsymbol{d}$ clearly illustrates that the critical spacing between an object and its closest neighbor (the flanker) depends also on the direction of the flankers with respect to the object.

tion performance at the peripheral retinal location adopted by people with macular lesions for seeing.

\section{Materials and Methods}

Observers. Eleven adults (aged 48-85 years, five males and six females) with long-standing bilateral macular lesions served as observers. Observers A-H had age-related macular degeneration, and observers I-K had Stargardt's disease (a type of juvenile macular degeneration). All had bilateral macular lesions for at least 3 years and had adapted to their condition by demonstrating a consistent preferred retinal locus (PRL) for a fixation task. The PRL refers to the location in the peripheral retina outside the lesioned foveal region that an individual with macular lesion adopts as the reference location for visual tasks (Cummings et al., 1985). The characteristics of these observers are given in Table 1. Testing was monocular using the preferred eye (usually the eye with better vision), or, when there was no preference, one of the two eyes was randomly selected for testing. Before data collection, the location of the PRL, fixation stability, visual acuity, and microperimetry (a technique for mapping the seeing and non-seeing regions of the retina) were performed in at least one previous session using the Rodenstock 101 scanning laser ophthal- moscope (SLO). Thus, all observers had some previous exposure with SLO testing. The SLO allowed us to image the retina while at the same time present stimuli at specific retinal locations.

For comparison, two groups of four older adults (aged 63-79 years, three males and five females) with normal vision in both eyes and unremarkable ocular health served as control observers. Crowding zones were measured at the fovea, $2.5^{\circ}, 5^{\circ}, 7.5^{\circ}$, and $10^{\circ}$ eccentricity in the nasal, lower, and lower-nasal visual fields for these control observers. Each group of control observers was tested at different retinal locations.

Stimuli. Stimuli were trigrams (sequences of three letters) rendered in lowercase Courier font. The middle letter of each trigram was designated as the target, to which observers verbally responded. All three letters of each trigram were randomly sampled from a rectangular distribution of the 26 letters, with no repetition allowed within a trigram. Trigrams were briefly presented (duration varied for different observers with macular lesions, from $667 \mathrm{~ms}$ to $1 \mathrm{~s}$ ) using the SLO, with the middle target letter centered at the PRL of each observer with macular lesions or at the desired retinal eccentricity (fovea, $2.5^{\circ}, 5^{\circ}, 7.5^{\circ}$, or $10^{\circ}$ nasal, lower, or lower-nasal visual field) for the older adult control observers. All letters were presented in their upright orientations regardless of the meridian of the crowding zone being evaluated.

For observers with macular lesions, letter sizes (referred to the $x$ height) in the main experiment ranged from $1^{\circ}$ to $3^{\circ}$, depending on the acuity of the observer. We repeated the testing with a few observers up to two more times after the original experiment and found that the shape of the crowding zone (critical spacings along several meridians) was fairly repeatable. For the control observers, letter sizes were $0.5^{\circ}$ at the fovea and $2.5^{\circ}$ eccentricity, $1^{\circ}$ at $5^{\circ}$ eccentricity, and $2^{\circ}$ at $7.5^{\circ}$ and $10^{\circ}$ eccentricity. Because of the spatial resolution of the SLO stimulus display, a letter size of $0.5^{\circ}$ was the smallest one that could be rendered on the display without the letters appearing too pixelated. Although this letter size could cause a floor effect of the smallest critical spacing that we could obtain [Toet and Levi (1992) reported a foveal critical spacing of $\left.\sim 0.05^{\circ}\right]$, the main point here is that the critical spacing is similar across different meridians at the fovea. In other words, the shape of the crowding zone in the fovea is isotropic. In the normal periphery, it is well known that the crowding zone is invariant of target size (Levi et al., 2002; Pelli et al., 2007; Levi and Carney, 2011). We performed additional testing using a larger letter size at $5^{\circ}$ and $10^{\circ}$ eccentricity for one group of control observers and indeed found that the shape of the crowding zone was similar for two letter sizes that differed by $1.5-2$ times at each eccentricity.

Apparatus. Stimuli were generated using the ViSaGe (Cambridge Research Systems) with custom-written software written in MATLAB 7.3.0 (MathWorks). Stimuli were presented on the raster display of the SLO. By interfacing the SLO video input and output signals using frame grabbers (Matrox Imaging Adapter: Meteor-II PCI Frame Grabber), retinal images were acquired at a frame rate of $30 \mathrm{~Hz}$ continuously for the duration of the entire trial (image capture started a few frames before and ended a few frames after the trial). The interface also allowed us to combine the stimulus signals with the video output of the retinal images for the recording. These video recordings were digitally processed offline, and the spatial locations corresponding to the target stimuli were determined by referencing the target locations with respect to the anatomical fovea.

Psychophysical methods. On each trial, a fixation cross the same size as the letters was first presented at the center of the raster display of the SLO. Observers were asked to maintain steady fixation on the cross. Testing was monocular. An experienced experimenter initiated a trial once the observer's fixation was deemed stable based on the fundus pictures captured by the SLO in real time, which then turned off the fixation cross. After a one-frame delay, the target letter, along with the two flanking letters, were presented simultaneously for 20 frames $(667 \mathrm{~ms})$ or 30 frames ( $1 \mathrm{~s})$, with the target letter occupying the original location of the fixation cross. The three letters, rendered in the upright orientation, were aligned with the center of each letter along the meridian being tested. The center-to-center separation between adjacent letters was varied according to an adaptive two-down-one-up staircase procedure. Observers were instructed to maintain fixation where the fixation cross was and to report whether or not they perceived three letters after each trial. If three 
Table 1. Visual characteristics of the 11 observers with macular lesions

\begin{tabular}{|c|c|c|c|c|c|c|c|c|c|c|}
\hline \multirow[b]{2}{*}{ Observer } & \multirow[b]{2}{*}{$M / F$} & \multirow[b]{2}{*}{ Age (years) } & \multirow[b]{2}{*}{ Diagnosis } & \multicolumn{2}{|c|}{ Acuity (logMAR) } & \multirow[b]{2}{*}{ Years since onset } & \multirow[b]{2}{*}{ PRL eccentricity $\left({ }^{\circ}\right)$} & \multirow[b]{2}{*}{ Letter size tested $\left({ }^{\circ}\right)$} & \multicolumn{2}{|l|}{ Fixation stability } \\
\hline & & & & OD & OS & & & & Horizontal SD $\left({ }^{\circ}\right)$ & Vertical SD $\left(^{\circ}\right)$ \\
\hline$A$ & $M$ & 84 & AMD & 0.56 & 0.70 & 8 & 6.16 & 1.5 & 1.35 & 0.27 \\
\hline$B$ & $\mathrm{~F}$ & 79 & AMD & 0.74 & 0.92 & 8 & 5.17 & 2 & 0.77 & 0.51 \\
\hline C & M & 84 & AMD & 0.44 & 0.50 & 19 & 2.45 & 1.5 & 0.52 & 0.18 \\
\hline D & $\mathrm{F}$ & 78 & AMD & 0.40 & 0.32 & 3 & 2.30 & 1 & 0.70 & 0.26 \\
\hline$E$ & M & 85 & AMD & 0.70 & 0.74 & 11 & 6.69 & 1.5 & 0.72 & 0.50 \\
\hline $\mathrm{F}$ & $\mathrm{F}$ & 82 & AMD & 0.50 & 0.52 & 9 & 3.49 & 1 & 0.32 & 0.33 \\
\hline G & $\mathrm{F}$ & 73 & AMD & 0.66 & 0.48 & 7 & 2.49 & 1.5 & 0.95 & 0.20 \\
\hline $\mathrm{H}$ & $\mathrm{F}$ & 74 & AMD & 0.54 & 1.12 & 6 & 1.19 & 1 & 0.18 & 0.08 \\
\hline I & M & 48 & Stargardt & 1.00 & 0.98 & 26 & 7.68 & 2 & 0.17 & 0.31 \\
\hline J & $M$ & 57 & Stargardt & 1.10 & 1.10 & 40 & 16.72 & 3 & 1.35 & 1.26 \\
\hline K & $\mathrm{F}$ & 62 & Stargardt & 0.58 & 0.58 & 29 & 2.63 & 1 & 1.20 & 0.76 \\
\hline
\end{tabular}

The tested eye is indicated by the acuity set in boldface. Fixation stability refers to the eye positions during trials of $30 \mathrm{~s}$ of fixation. AMD, Age-related macular degeneration; $F$, females; $M$, males; $0 \mathrm{D}$, right eye; $0 \mathrm{~S}$, left eye; logMAR, logarithm of the minimum angle of resolution.

letters were perceived, then observers were asked to verbally report the identity of the middle letter. Trials for which observers did not perceive three letters were discarded. Feedback was not provided after each response. Trials were also discarded if observers made large eye movements during testing such that the target letter was imaged on a location away from the PRL. However, this only happened occasionally. In general, observers were able to maintain a relatively stable PRL throughout testing. The staircase terminated when the 10th reversal was reached. Separate staircases were used to measure the threshold critical spacing along four different meridians: $0^{\circ}$ (horizontal), $45^{\circ}, 90^{\circ}$ (vertical), and $135^{\circ}$ (Fig. $2 a$ ).

Data analyses. All data analyses were performed offline.

Determining the threshold critical spacing. Video recording of all trials were reviewed to ensure that observers did not miss a trial because of an eyeblink or a large eye movement. Also, trials in which one or more letters fell within the scotoma (determined previously using microperimetry) and that the observer did not report to the experimenter during testing were excluded for analysis. Then, for trials collected during a given staircase, we constructed a psychometric function relating the identification accuracy with letter separation. A cumulative Gaussian function was used to fit each set of data, and the letter separation corresponding to $52 \%$ correct [half-way between chance ( 1 of 26 ) and perfect performance] on the psychometric function was defined as the threshold critical spacing for crowding.

Determining the location of the anatomical fovea and the PRL. For observers with macular lesions, the location of the anatomical fovea was determined with respect to the center of the optic nerve head. Therefore, it did not matter whether the anatomical fovea was clearly defined or visible on the recorded retinal images. For each SLO video, we randomly extracted at least 60 frames that contained clear images of the optic nerve head for analysis. For each frame, a minimum of five points around the edge of the optic nerve head were located, and an ellipse was fit to the set of points using MATLAB. The coordinates of the center of the fitted ellipse were determined. Based on previous empirical measurements obtained in our laboratory from 18 young and 18 older adults, we determined that the distance between the anatomical fovea and the center of the optic nerve head averages $15.12 \pm 1.04^{\circ}$ (range, $\left.12.84-17.36^{\circ}\right)$ horizontally and $-1.37 \pm 0.84^{\circ}$ (range, $-3.47^{\circ}$ to $0.48^{\circ}$ ) vertically (the anatomical fovea is below the center of the optic nerve head in most eyes). These measurements, including the individual variations and ranges, were highly comparable with similar data reported by other laboratories, with sample sizes of 50-104 eyes (Rohrschneider, 2004; Timberlake et al., 2005). We then used this set of "standard" distances to specify the spatial location of the PRL with respect to the anatomical fovea. Although there is an uncertainty (error) associated with using a set of standard distances to locate the anatomical fovea, and thus the PRL, the uncertainty, represented by the standard deviation of the estimate, is $\sim 1^{\circ}$. As we shall see later, the main finding of the study relates to the shape of the crowding zone, which does not directly depend on the exact eccentricity. Therefore, a small error in locating the PRL is unlikely to affect our main finding.

Fitting ellipses to describe crowding zones. The threshold spacing determined along the four meridians $\left(0^{\circ}, 45^{\circ}, 90^{\circ}\right.$, and $\left.135^{\circ}\right)$ at each testing location was fit by an ellipse using MATLAB, from which the magnitudes of the major and minor axes, and the orientation of the ellipse (defined as the angle of the major axis from horizontal in the counterclockwise direction), were derived. This process was repeated 1000 times using bootstrap resampling so that we could obtain an estimate of the error (represented by the SD of the distribution) associated with each parameter (major axis, minor axis, and the orientation of the ellipse). Each ellipse shown in Figure 2 was reconstructed from the average value of each parameter derived from the bootstrap resampling. Based on the location of the PRL relative to the anatomical fovea, we identified the radial and tangential axes of the crowding zone. The critical spacings along these radial and tangential axes were then calculated.

\section{Results}

We first mapped the two-dimensional crowding zone at the fovea and from $2.5^{\circ}$ to $10^{\circ}$ eccentricity in the nasal, lower, and lowernasal visual fields of older adults with normal vision. The results are plotted as gray symbols and ellipses in Figure 2, $c$ and $d$. Each symbol represents the critical spacing measured from a tested retinal location (plotted as a + ) along a given meridian, averaged across four older adults with normal vision. The ellipse around each set of eight symbols represents the average best-fit ellipse, with the major and minor axes and the orientation of the ellipse derived from 1000 bootstrap resamplings. In the normal fovea, the critical spacing is much smaller than in the periphery, and the magnitude is similar along the various meridians, confirming that the shape of the crowding zone at the fovea is isotropic (Toet and Levi, 1992). In the normal periphery, there is a substantial difference in the magnitude of the critical spacing depending on the meridian, with the largest critical spacing oriented radially toward the fovea and the smallest one tangential to the fovea, such that there is a substantial radial-tangential anisotropy (the shape of the crowding zone being anisotropic; Toet and Levi, 1992; Pelli et al., 2007; Pelli, 2008). The size of the crowding zone also progressively increases with eccentricity. The radial (tangential) dimension of the crowding zones increased from $1.27^{\circ}$ $\left(0.84^{\circ}\right)$ at $2.5^{\circ}$ eccentricity to $4.58^{\circ}\left(2.77^{\circ}\right)$ at $10^{\circ}$ eccentricity. In other words, the radial (tangential) size of the crowding zone approximates 0.48 times ( 0.30 times) the eccentricity, approximately consistent with Bouma's law and previous studies (Toet and Levi, 1992; Chung et al., 2001; Pelli et al., 2007; Pelli, 2008). These findings from the normal fovea and periphery validated our experimental paradigm to be used to determine the shape and size of the crowding zones at the PRL of individuals with long-standing macular lesions.

To quantify the anisotropy (or the lack thereof) of the shape of the crowding zone, at each retinal location, we fit the critical 
a

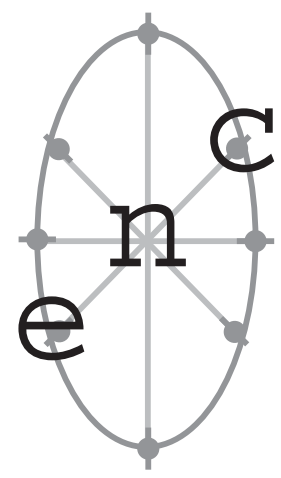

b
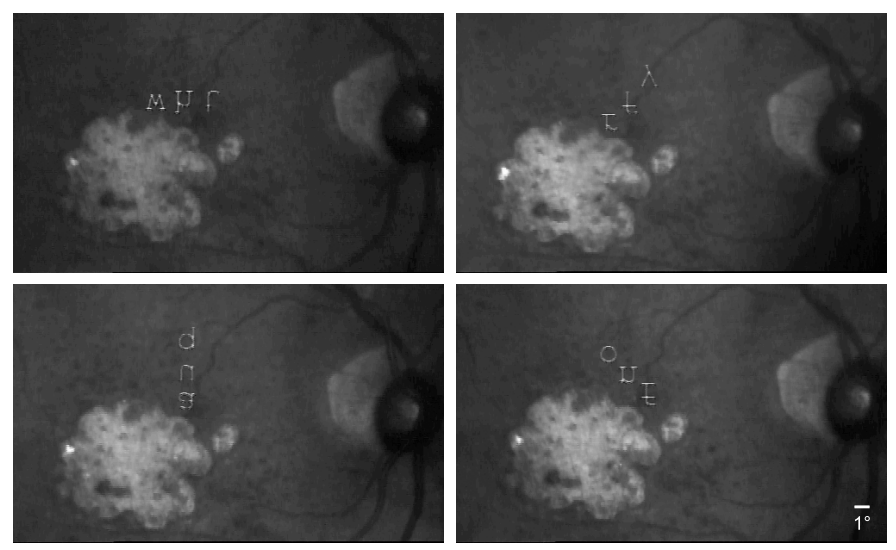

C

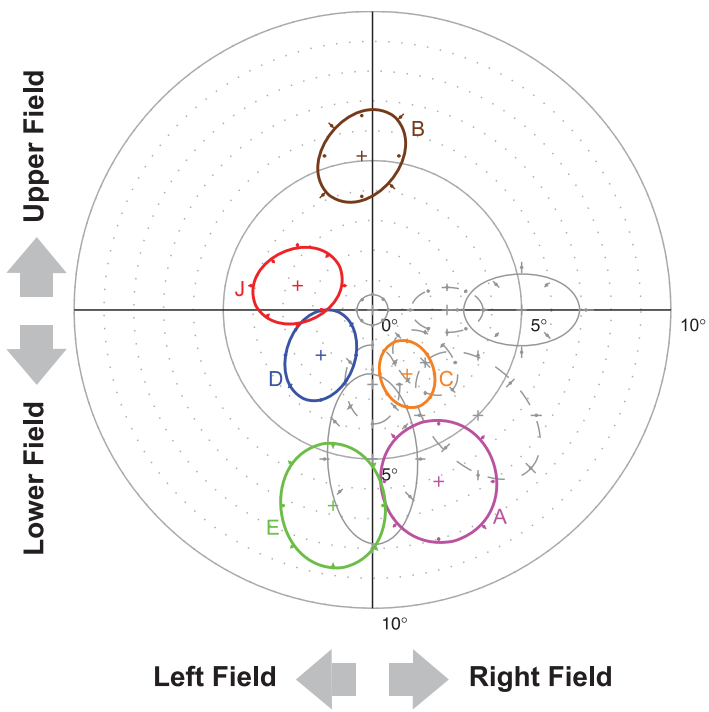

d

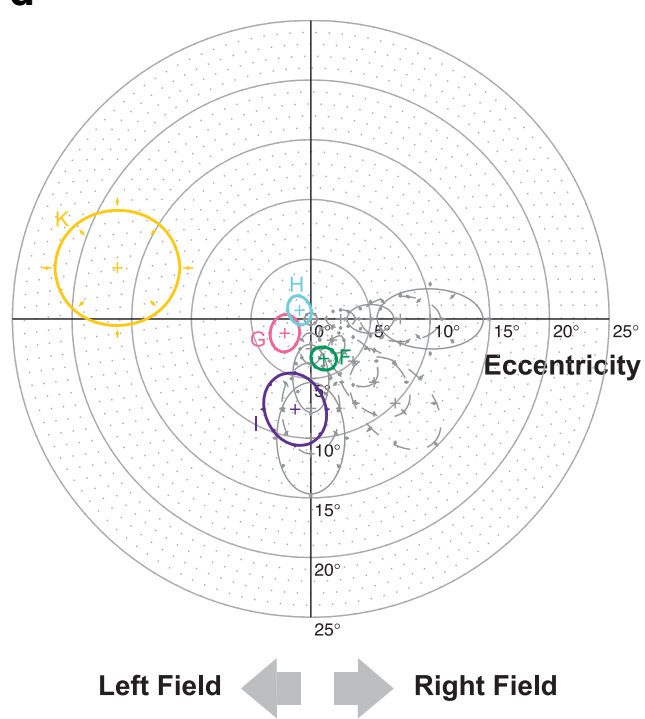

Figure 2. $a$, A schematic figure showing that the critical spacing was determined along four meridians $\left(0^{\circ}, 45^{\circ}, 90^{\circ}\right.$, and $135^{\circ}$ from horizontal), and an ellipse was fit to the set of data. For illustration purpose, three letters, $c, n$, and e, are presented along the $45^{\circ}$ meridian, with $n$ being the target letter and is shown at the intended retinal location for testing. 0 bservers verbally reported the identity of the target letter after the offset of the three letters. The spacing between letters that yielded $52 \%$ correct on the psychometric function [half-way between chance ( 1 of 26 ) and perfect performance] was defined as the threshold critical spacing (represented by the gray circles). $\boldsymbol{b}$, Samples of the pictures of the retina of observer F showing trials in which the three letters of a trigram were oriented along the horizontal (top left), $45^{\circ}$ diagonal (top right), vertical (bottom left), and $135^{\circ}$ diagonal (bottom right) meridians, at locations close to the lesioned areas (the lighter colored regions, representing the non-seeing scotoma when projected in the visual field domain). These samples were extracted directly from the video recording of several experimental trials in which the stimulus letters were combined with the pictures of the retina before recording, thus explaining the quality of the letters. In reality, the letters were much clearer when presented to observers during testing. The middle letter of each trigram was the target letter to which observers were asked to verbally respond. The averaged retinal location corresponding to the center of the target letter was used to represent the PRL. Although the letters were displayed as upside down in the figure, observers saw the letters as upright within the SLO. The scale bar is given in the bottom right corner of the bottom right panel. c, A polar plot showing the two-dimensional shapes of the crowding zones for six observers with macular lesions (observers A-E and J). The threshold critical spacings for each observer are plotted as different colored symbols. Error bars represent $\pm 1 \mathrm{SE}$ for these data. We used bootstrap resampling to fit an ellipse to each set of data from which we derived the major and minor axis and the orientation of the ellipse. Each ellipse shown here was reconstructed based on the average value of these parameters after 1000 resamplings. For comparison, the crowding zones obtained at the fovea (center of the plot) and at $2.5^{\circ}, 5^{\circ}, 7.5^{\circ}$, and $10^{\circ}$ in the nasal (plotted as the right field in the figure), lower, and lower-nasal visual fields of older adults with normal vision (control) are shown in gray. Solid and dashed ellipses represent data from the two groups of control older adults (4 in each group). For the control observers, error bars represent \pm 1 SD of the group values. Each crowding zone is centered at the PRL of an observer with macular lesions or at the intended retinal location for the control observers. $\boldsymbol{d}$, A second polar plot showing the two-dimensional shapes of the crowding zones for the other five observers with macular lesions (observers $\mathrm{F}-\mathrm{I}$ and $\mathrm{K}$ ). Details of the plot are the same as for $\mathrm{c}$. Note the change in scale of the plot.

spacing measured along the four meridians (eight points altogether) and for the four control observers with an ellipse. We used the bootstrap resampling technique to resample each set of data 1000 times, from which we obtained the average magnitude of the major axis, minor axis, and the orientation of the ellipse. The ellipses shown in gray in Figure 2, $c$ and $d$, represent the ellipses reconstructed based on the parameters obtained from bootstrap resampling. For each of these ellipses, we calculated the critical spacing along the radial and tangential axis. The ratio of the critical spacing between the radial and tangential axis, the anisotropy index, was used to represent the two-dimensional shape of the crowding zone. An anisotropy index of $1 \mathrm{implies}$ that the shape of the crowding zone is circular and isotropic. The anisotropy indices for the crowding zone were 1.02 at the fovea and averaged $1.62 \pm 0.07$ (95\% confidence intervals) across the 12 tested locations in the periphery (no systematic differences across eccentricities or for the different visual fields). The anisotropy index for the crowding zones in the normal periphery is consistent with estimated values reported in the literature ( 1.5-2.5: Toet and Levi, 1992; Pelli et al., 2007; Nandy and Tjan, 2012). 
Next we determined the PRL for the 11 human observers who lost their macula in their fourth to eighth decade of life by identifying the retinal locations the observers used to look at visual stimuli. Across observers, the PRLs were located between $1.2^{\circ}$ and $16.7^{\circ}$ from the fovea $\left(\right.$ median of $\left.3.5^{\circ}\right)$. We then measured the crowding zone centered on each observer's PRL by imaging the retina and presenting the visual stimuli at precise retinal locations with respect to the PRL (for sample trials showing the stimulus superimposed on the retina of observer $\mathrm{F}$ along the four tested meridians, see Fig. $2 b$ ). The two-dimensional crowding zones for these 11 observers with macular lesions, as described by the fitted ellipses, are plotted in different colors in Figure 2, $c$ and $d$. Contrary to the predictions based on the normal periphery in which the radial-tangential anisotropy is a signature property of the crowding zone, the crowding zones for observers with macular lesions show less of a radial-tangential anisotropy. Across the group of observers, there was no difference between the orientation of the major (minor) axis of the fitted ellipse and the radial (tangential) meridian of the crowding zone (paired $t$ test, $t_{\mathrm{df}}=10=$ $1.37 ; p=0.20$ ). The anisotropy indices thus derived based on the radial and tangential meridians of the fitted ellipses ranged from 0.93 to 1.27 [mean of $1.08 \pm 0.06$ ( $95 \%$ confidence intervals)] significantly smaller than what we observed in the normal periphery of our control group (two-tailed, two-sampled $t$ test with unequal variance, $\left.t_{\mathrm{df}}=20.4=8.24 ; p<0.0001\right)$. This finding clearly shows that, contrary to our understanding of the normal periphery (Pelli, 2008; Pelli and Tillman, 2008), the critical spacing at a peripheral retinal location is not a constant value determined solely by the retinal location but can be modified after long-term adaptation to macular lesions. More importantly, the 95\% confidence intervals of the anisotropy indices for observers with macular lesions include the value obtained for the normal fovea (1.02), implying that the shape of the crowding zone at the PRL for our group of observers with macular lesions is not different from that of the normal fovea. To our knowledge, this is the first time that a property of a peripheral retinal location in humans with retinal lesions has been shown to resemble more closely that of the normal fovea instead of the normal periphery.

Although we did not have measurements of the crowding zones at the PRL locations for the 11 observers with macular lesions before the onset of their lesions, it is reasonable to assume that the crowding zones at the eccentric retinal locations corresponding to the eventual location of the PRL were anisotropic in shape before the onset of macular lesions, as expected based on the normal periphery. To further characterize this loss of anisotropy of the crowding zone after the onset of macular lesions, we compared the critical spacing along the radial and tangential axes of the crowding zones for observers with macular lesions with those of the normal periphery. We found that the radial axis of the crowding zones for observers with macular lesions were substantially smaller than the predictions based on the normal periphery, when the eccentricities of the PRL locations were
Radial Axis

Tangential Axis

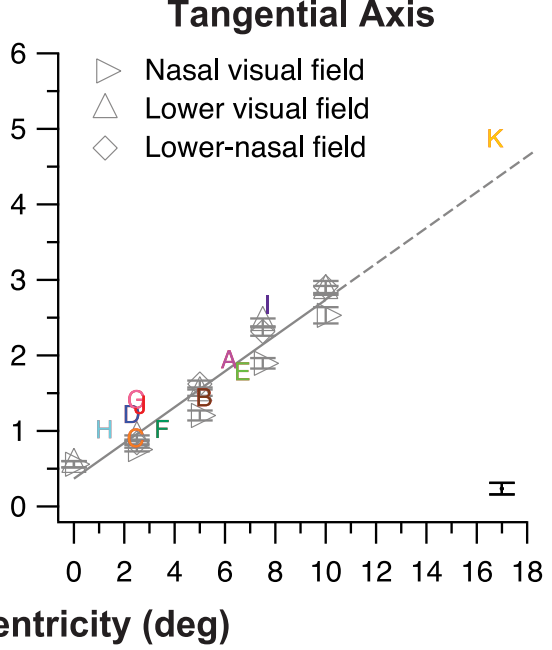

Retinal Eccentricity (deg)

Figure 3. Critical spacing (in degrees) along the radial and tangential axes of the fitted ellipses as shown in Figure 2, c and d, are among the 11 observers is shown here, plotted as the error bar in the bottom right corner of each panel. For comparison, the critical in each panel represents the best-fit regression line to the data of the control observers and is extended (shown in dashes)

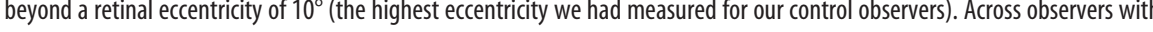
However, the critical spacing along the radial axis is smaller than the predicted value based entirely on the retinal eccentricity for

matched (Fig. 3). In contrast, the tangential axis of the crowding zones for observers with macular lesions were similar in magnitude to the predictions based on the normal periphery. The smaller values of the critical spacing along the radial axis found for observers with macular lesions, compared with the normal periphery, implies that the reduction in the radial-tangential anisotropy of the crowding zone at the PRL of observers with macular lesions resulted from a shrinkage of the critical spacing along the radial axis rather than an expansion along the tangential axis.

\section{Discussion}

Our main finding is that the crowding zone, the neuroanatomical limitation of object recognition, at the PRL of people with longstanding macular lesions exhibits a loss of the radial-tangential anisotropy that is the signature of the normal periphery. The reduction in the anisotropy is attributable to shrinkage of the radial axis of the crowding zone at the PRL, resulting in the shape of the crowding zone resembling that of the fovea rather than the periphery. We postulate that these changes are caused by the repeated usage of the PRL for visual tasks for years after the macular lesions. However, we distinguish these changes from the changes in task performance that follow a period of intensive training on a perceptual task (perceptual learning). Visual performance is known to improve with perceptual learning, in which observers repeatedly practice the same task with similar stimulus configuration intensively for a relatively short period of time. The improvements usually occur fairly quickly; for instance, a reduction in the critical spacing was observed after only $6-12 \mathrm{~h}$ of intensive training (Chung, 2007; Chung et al., 2012; Hussain et al., 2012). Our observers did not receive intensive training for any specific type of visual task; therefore, the observed changes could not be attributed to perceptual learning but instead are likely to be attributable to the repeated usage of the PRL for a variety of 


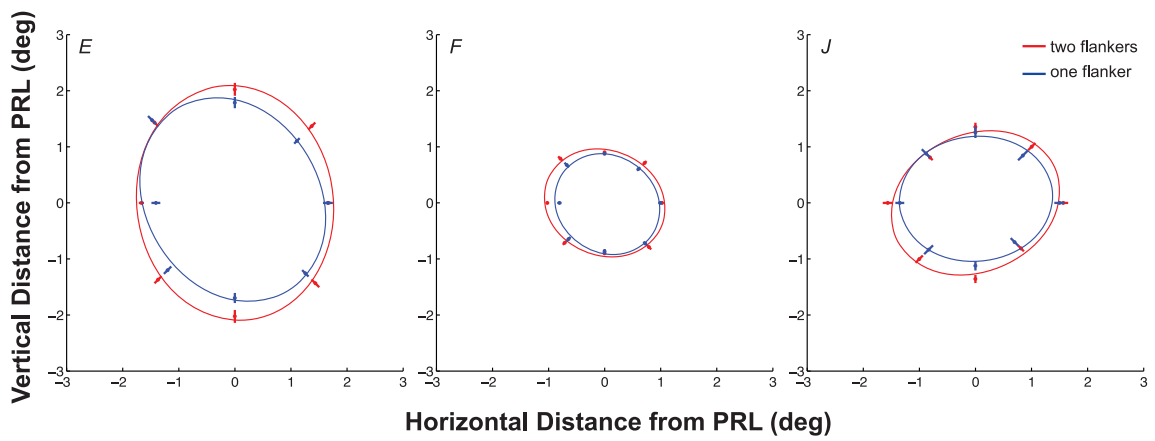

Figure 4. The critical spacings along eight meridians, representing the two-dimensional crowding zone, are compared between two conditions for three observers with macular lesions (observers E, F, and J). The two conditions were as follows: (1) two flankers were presented along a given meridian and the distance between either one of them and the target was yoked (the main experiment); and (2) only one flanker was presented. Each set of eight critical spacings was fit with an ellipse. The center of each panel $[$ coordinate $(0,0)]$ represents the location of the PRL. Error bars represent $\pm 1 \mathrm{SE}$ of the estimate of the critical spacing.

Here, we found that the radial axis of the crowding zone at the PRL is reduced in size. Therefore, even if the receptive fields outside the border of the macular lesion were enlarged, it would not be able to account for the shrinkage in the critical spacing along the radial axis.

Individuals with macular lesions are known to have poor fixation stability (for the variability in eye positions during trials of 30 s of fixation, see Table 1), conventionally quantified by fitting an ellipse to the eye positions during a short period of fixation. It is reasonable to expect that unstable fixation along a given meridian might lead to a larger critical spacing along the same meridian. Using an ellipse to describe the eye positions during fixa-

visual tasks over the years. Nevertheless, the repeated usage of the PRL alone cannot readily explain why the reduction in the critical spacing is found only along the radial direction instead of along all directions of the crowding zone.

Can our result be attributed to the flanker closer to the nonseeing lesioned area (the inner flanker) falling within the region, thus weakening the crowding effect? This is unlikely because we took several precautions to ensure that all the data reported here were collected from trials in which observers saw all three letters. Trials were discarded online if observers reported not seeing all three letters or when the experimenter saw that one flanker fell within the scotoma. Trials were also discarded offline before data analysis when the experimenter reviewed all the video recordings. Nevertheless, as a control experiment, we remapped the crowding zone at the PRL of three observers with macular lesions using only one flanker at a time. The measurements of the critical spacing are generally more variable when only one flanker was presented (Petrov and Meleshkevich, 2011; Nandy and Tjan, 2012). Considering the larger variability in measurements, Figure 4 shows that the crowding zones obtained using one flanker are slightly smaller than those obtained using two flankers, but the anisotropy index between the radial and tangential axes remains similar [two flankers vs one flanker: 1.15 vs 1.02 (observer E), 0.98 vs 1.03 (observer F), 1.03 vs 1.15 (observer J)], implying that the reduced anisotropy of the crowding zone at the PRL cannot be attributed to one flanker falling within the scotoma.

Although observers saw all three letters, perhaps the inner flankers, being closer to the lesioned area that might not be healthy, were less visible and thus weakened the crowding effect? Pelli et al. (2004) show that, as soon as a flanker is detectable, its visibility does not affect the critical spacing. Therefore, even if the inner flankers were less visible, it would not have affected the critical spacing.

Seminal work using single-unit recording in cats and monkeys has shown that, after bilateral retinal lesions that abolish visual input to the cortical areas, the receptive fields of neurons close to the edge of the lesion projection zone could become larger within minutes of the lesions (Kaas et al., 1990; Heinen and Skavenski, 1991; Chino et al., 1992; Gilbert and Wiesel, 1992) and thus can respond to visual stimulus falling inside but close to the border of the lesion area. Could the enlargement of the receptive field in response to retinal lesions account for our result, for instance, via long-range interactions? It is reasonable to expect that a larger receptive field would lead to more interference between visual stimuli, thus leading to a larger but not a smaller crowding zone. tion of our 11 observers with macular lesions, we found that neither the major axis of the fixation ellipse correlates with the major axis of the crowding zone, nor the area of the fixation ellipse correlates with the area of the ellipse that describes the crowding zone. These findings rule out fixation instability as contributing to the isotropic shape of the crowding zone at the PRL.

A recent theory attributes the anisotropy of the crowding zone in the normal periphery to saccade-confounded image statistics because normal saccadic eye movements are radial with respect to the fovea (Nandy and Tjan, 2012). This theory predicts that, as the PRL becomes the oculomotor reference for people with macular lesions, saccades will be directed to the PRL instead of the fovea; consequently, the visual system will be exposed to a new set of image statistics at the PRL unconfounded by the radial image motion toward the fovea. With time, the visual system relearns this new set of image statistics that contains less overintegration of information as a direct result of the radial retinal image motion toward the fovea, thus reducing the radial-tangential anisotropy at the PRL. Accordingly, if the oculomotor re-referencing process is complete, there should be a complete lack of anisotropy of the crowding zone at the PRL. Evidence that people with longstanding macular lesions execute saccades toward the PRL comes from White and Bedell (1990) and Whittaker et al. (1991), although in most cases, the re-referencing was incomplete. Unpublished data from our laboratory show that the degree of completeness of the oculomotor re-referencing process for 10 of our observers with macular lesions ranged from 66.8 to $94.3 \%$. These high degrees of completeness of the oculomotor rereferencing are consistent with the close-to-one anisotropy indices observed for our observers (range of 0.93-1.27).

Animal studies have established that neurons around the lesion projection zone in adult visual cortex could respond to visual stimuli fallen within the non-seeing areas. Attempts at establishing the evidence for this type of cortical reorganization in adult humans with retinal lesions have yielded equivocal results (Sunness et al., 2004; Baker et al., 2005, 2008; Masuda et al., 2008; Schumacher et al., 2008; Dilks et al., 2009; Liu et al., 2010; Baseler et al., 2011). These studies used fMRI to measure cortical activities and the changes in BOLD signals to represent whether there are visual signals originated from within the lesioned areas. Our study is different in that we focus on the changes at a specific location outside the lesioned areas (the PRL), using observers' behavioral performance as evidence of cortical plasticity. Our findings imply a unique form of cortical reorganization, perhaps not anatomical but one that, based on visual experience, modifies 
the representation of visual information in early sensory areas of the brain that correspond to the location of the PRL and/or the read-out process.

Where in the brain does this form of cortical reorganization occur? Our finding suggests that the neural site of crowding may provide the answer to this question. By comparing changes in the receptive field size in different visual areas and the size of crowding zone with eccentricity, the cortical substrate for crowding has been placed at V1 (Nandy and Tjan, 2012), V2 (Freeman and Simoncelli, 2011), V3 (Tyler and Likova, 2007; Bi et al., 2009), V4 (Motter, 2006), or higher (Chung et al., 2007). The wide range of suggested neural sites for crowding is not surprising given that crowding can occur at multiple stages in the visual hierarchy (Whitney and Levi, 2011) and has been attributed to different mechanisms, from faulty feature integration in V1 to high-level attentional deficits. Thus, the form of reorganization we postulated could occur as early as in V1, which would be consistent with an experience-based modification of the sensory coding of stimulus, but the observed changes in the crowding zone can also represent the feedback of neuronal influences attributable to reorganization at higher cortical areas or simply the effect of attention. Future behavioral studies using stimuli designed to specifically stimulate certain visual areas will be useful in identifying the exact locus of this form of cortical reorganization.

\section{References}

Baker CI, Peli E, Knouf N, Kanwisher NG (2005) Reorganization of visual processing in macular degeneration. J Neurosci 25:614-618. CrossRef Medline

Baker CI, Dilks DD, Peli E, Kanwisher N (2008) Reorganization of visual processing in macular degeneration: replication and clues about the role of foveal loss. Vision Res 48:1910-1919. CrossRef Medline

Baseler HA, Gouws A, Haak KV, Racey C, Crossland MD, Tufail A, Rubin GS, Cornelissen FW, Morland AB (2011) Large-scale remapping of visual cortex is absent in adult humans with macular degeneration. Nat Neurosci 14:649-655. CrossRef Medline

Bi T, Cai P, Zhou T, Fang F (2009) The effect of crowding on orientationselective adaptation in human early visual cortex. J Vis 9(11):13 1-10. CrossRef

Bouma H (1970) Interaction effects in parafoveal letter recognition. Nature 226:177-178. CrossRef Medline

Chino YM, Kaas JH, Smith EL 3rd, Langston AL, Cheng H (1992) Rapid reorganization of cortical maps in adult cats following restricted deafferentation in retina. Vision Res 32:789-796. CrossRef Medline

Chung STL (2007) Learning to identify crowded letters: does it improve reading speed? Vision Res 47:3150-3159. CrossRef Medline

Chung STL, Levi DM, Legge GE (2001) Spatial-frequency and contrast properties of crowding. Vision Res 41:1833-1850. CrossRef Medline

Chung STL, Li RW, Levi DM (2007) Crowding between first- and secondorder letter stimuli in normal foveal and peripheral vision. J Vis 7(2):10 1-13. CrossRef

Chung STL, Li RW, Levi DM (2012) Learning to identify near-acuity letters, either with or without flankers, results in improved letter size and spacing limits in adults with amblyopia. PLoS One 7:e35829. CrossRef Medline

Cummings RW, Whittaker SG, Watson GR, Budd JM (1985) Scanning characters and reading with a central scotoma. Am J Optom Physiol Opt 62:833-843. CrossRef Medline

Dilks DD, Baker CI, Peli E, Kanwisher N (2009) Reorganization of visual processing in macular degeneration is not specific to the "preferred retinal locus." J Neurosci 29:2768-2773. CrossRef

Flom MC, Heath GG, Takahashi E (1963) Contour interaction and visual resolution: contralateral effect. Science 142:979-980. CrossRef Medline

Freeman J, Simoncelli EP (2011) Metamers of the ventral stream. Nat Neurosci 14:1195-1201. CrossRef Medline
Gilbert CD, Wiesel TN (1992) Receptive field dynamics in adult primary visual cortex. Nature 356:150-152. CrossRef Medline

Heinen SJ, Skavenski AA (1991) Recovery of visual responses in foveal V1 neurons following bilateral foveal lesions in adult monkey. Exp Brain Res 83:670-674. Medline

Hussain Z, Webb BS, Astle AT, McGraw PV (2012) Perceptual learning reduces crowding in amblyopia and in the normal periphery. J Neurosci 32:474-480. CrossRef Medline

Kaas JH, Krubitzer LA, Chino YM, Langston AL, Polley EH, Blair N (1990) Reorganization of retinotopic cortical maps in adult mammals after lesions of the retina. Science 248:229-231. CrossRef Medline

Levi DM (2008) Crowding—an essential bottleneck for object recognition: a mini-review. Vision Res 48:635-654. CrossRef Medline

Levi DM, Carney T (2011) The effect of flankers on three tasks in central, peripheral, and amblyopic vision. J Vis 11(1):10 1-23. CrossRef Medline

Levi DM, Hariharan S, Klein SA (2002) Suppressive and facilitatory spatial interactions in peripheral vision: peripheral crowding is neither size invariant nor simple contrast masking. J Vis 2:167-177. CrossRef Medline

Liu T, Cheung SH, Schuchard RA, Glielmi CB, Hu X, He S, Legge GE (2010) Incomplete cortical reorganization in macular degeneration. Invest Ophthalmol Vis Sci 51:6826-6834. CrossRef Medline

Masuda Y, Dumoulin SO, Nakadomari S, Wandell BA (2008) V1 projection zone signals in human macular degeneration depend on task, not stimulus. Cereb Cortex 18:2483-2493. CrossRef Medline

Motter BC (2006) Modulation of transient and sustained response components of V4 neurons by temporal crowding in flashed stimulus sequences. J Neurosci 26:9683-9694. CrossRef Medline

Nandy AS, Tjan BS (2012) Saccade-confounded image statistics explain visual crowding. Nat Neurosci 15:463-469. CrossRef Medline

Pelli DG (2008) Crowding: a cortical constraint on object recognition. Curr Opin Neurobiol 18:445-451. CrossRef Medline

Pelli DG, Tillman KA (2008) The uncrowded window of object recognition Nat Neurosci [Erratum (2008) 11:1463] 11:1129-1135. CrossRef Medline

Pelli DG, Palomares M, Majaj NJ (2004) Crowding is unlike ordinary masking: distinguishing feature integration from detection. J Vis 4:1136-1169. CrossRef Medline

Pelli DG, Tillman KA, Freeman J, Su M, Berger TD, Majaj NJ (2007) Crowding and eccentricity determine reading rate. J Vis 7(2):20 1-36. CrossRef

Petrov Y, Meleshkevich O (2011) Asymmetries and idiosyncratic hot spots in crowding. Vision Res 51:1117-1123. CrossRef Medline

Rohrschneider K (2004) Determination of the location of the fovea on the fundus. Invest Ophthalmol Vis Sci 45:3257-3258. CrossRef Medline

Schumacher EH, Jacko JA, Primo SA, Main KL, Moloney KP, Kinzel EN, Ginn J (2008) Reorganization of visual processing is related to eccentric viewing in patients with macular degeneration. Restor Neurol Neurosci 26:391-402. Medline

Sunness JS, Liu T, Yantis S (2004) Retinotopic mapping of the visual cortex using functional magnetic resonance imaging in a patient with central scotomas from atrophic macular degeneration. Ophthalmology 111: 1595-1598. CrossRef Medline

Timberlake GT, Sharma MK, Grose SA, Gobert DV, Gauch JM, Maino JH (2005) Retinal location of the preferred retinal locus relative to the fovea in scanning laser ophthalmoscope images. Optom Vis Sci 82:177-185. Medline

Toet A, Levi DM (1992) The two-dimensional shape of spatial interaction zones in the parafovea. Vision Res 32:1349-1357. CrossRef Medline

Tyler CW, Likova LT (2007) Crowding: a neuroanalytic approach. J Vis 7(2):16 1-9. CrossRef

White JM, Bedell HE (1990) The oculomotor reference in humans with bilateral macular disease. Invest Ophthalmol Vis Sci 31:1149-1161. Medline

Whitney D, Levi DM (2011) Visual crowding: a fundamental limit on conscious perception and object recognition. Trends Cogn Sci 15:160-168. CrossRef Medline

Whittaker SG, Cummings RW, Swieson LR (1991) Saccade control without a fovea. Vision Res 31:2209-2218. CrossRef Medline 